Tohoku J. exp. Med., 1983, 141, Suppl., 523-528

\title{
Trend of Macroangiopathy in Diabetics Studied by Postmortum Examination
}

\author{
Hiroshi Takekoshi, Shin-ichi Kitamura, Kempei \\ Matsuoka, Yoshinito Atsumi, Akira Horiuchi, \\ Toshinaru Ishit*, Yasuhiro Hosoda*, Seiya Akatsuka \\ and Younosuke Watanabe*
}

Department of Internal Medicine, Saiseikai Central
Hospital, Tokyo and ${ }^{*}$ Department of Pathology Keio
University School of Medicine Tokyo, Tokyo 160, Japan

Takekoshi, H., Kitamura, S., Matsuoka, K., Atsumi, Y., Horiuchi, A., Iseir, T., Hosoda, Y., Akatsuka, S. and Watanabe, Y. Trend of Macroangio pathy in Diabetics Studied by Postmortum Examination. Tohoku J. exp. Med., 1983, 141, Suppl., 523-528_— In recent years, both a numerical and relative increase in the morbidity of cardiovascular and cerebral vascular diseases has been noted in Japanese diabetics. The trend of macroangiopathy in the period between 1953 and 1980 was studied in 210 autopsy cases (Group A : 1953-62, 27 cases, Group B : 1963-72, 43 cases and Group C : 1973-80, 140 cases). The incidence of myocardial infarction and cerebral infarction as a primary cause of death was 25 . $9 \%$ in Group A, 23.3\% in Group B and $37.9 \%$ in Group C. From the autopsy findings, the morbidity of cardiovascular disease was $14.8 \%, 30.2 \%$ and $34.3 \%$ respectively. In order to elucidate the clinical risk factors, the age at death, duration of diabetes at the time of death, and incidence of hypertension and serum cholesterol in the past history were studied. The percentages of those aged over 70 at death was $18.5 \%, 23.3 \%$ and $42.8 \%$ respectively, and those of the cases with durations of more than 10 years was $25.9 \%, 40.5 \%$ and $51.8 \%$ respectively. The prevalence of hypertension and hypercholesterolemia was almost identical in each group. From the above, the previous increase in the death-rate due to coronary heart disease and cerebrovascular disease in Japanese diabetics is thought to be attributable to the increasing number of elderly diabetics of long duration. —_ macroangiopathy in diabetes mellitus; autopsy findings; cerebrovascular disease; cardiovascular disease

In the previous three decades, both a numerical and relative increase in morbidity of cardiovascular and cerebrovascular disease has become increasingly noticeable among diabetics in Japan. This study is aimed at elucidating the chronological trend in the cause of death in an urban area of Japan through autopsy findings in diabetics at Saiseikai Central Hospital, Tokyo.

\section{Materials and Method}

The autopsy records of 210 diabetics (male 151, female 59) who died between 1953 and 80 were reviewed. (Table 1). As a reference group, the autopsy records of 743 non-diabetic 
subjects who died in the same period were also reviewed. The subjects of both groups were chronologically classified into 3 groups, Group A, 27 diabetics and 44 non-diabetics in 195362, Group B, 43 diabetics and 315 non-diabetics in 1963-72 and Group C, 140 diabetics and 305 non-diabetics in 1973-80. To evaluate the clinical course, the age at death, sex, duration of diabetes, obese ratio, glycemic control, serum total cholesterol, blood pressure and so on were studied. Through each clinical course, body weight more than $10 \%$ in excess of the standard weight recorded for more than one third of the observation period was determined as obesity; glycemic control was determined by fasting blood glucose values in two thirds of the test results; hypercholesterolemia was determined by $230 \mathrm{mg} / 100 \mathrm{ml}$ or more in one third of the test results; and hypertension was determined by a blood pressure over $160 \mathrm{mmHg}$ systole or $95 \mathrm{mmHg}$ diastole. The statistical significance was calculated by the chi square test.

TABLE 1. Autopsied diabetic subjects

\begin{tabular}{|c|c|c|c|c|}
\hline \multirow[b]{2}{*}{ Sex } & \multicolumn{3}{|c|}{ Year } & \multirow{2}{*}{$\begin{array}{l}\text { Total } \\
\text { (cases) }\end{array}$} \\
\hline & $1953 \sim 62$ & $1963-72$ & $1973 \sim 80$ & \\
\hline Male & 17 & 36 & 98 & 151 \\
\hline Female & 10 & 7 & 42 & 59 \\
\hline Total & 27 & 43 & 140 & 210 \\
\hline
\end{tabular}

\section{RESULTS}

The chronological trend for primary causes of death in diabetics is shown in Table 2. Prevalence of death due to macroangiopathies was $25.9 \%$ in Group A, $23.3 \%$ in Group B and $37.9 \%$ in Group C. A remarkable increase in the ratio of death due to myocardial infarction was demonstrated with values of $7.4 \%, 9.3 \%$ and $18.6 \%$ respectively, while cerebrovascular diseases remained unchanged. Death from malignancy increased but infectious causes showed a remarkable decrease in previous years.

In the non-diabetic reference groups, the death ratio due to malignancy was $12.5 \%$ in Group A, 35.2\% in Group B and $47.2 \%$ in Group C, and the ratios due to infectious disease were $29.9 \%, 15.2 \%$ and $9.5 \%$ respectively. (Table 3 ). Among macroangiopathic diseases, our findings show a gradual decrease in death due to cerebral hemorrhage. Cerebral infarction (CI) remained unchanged and myocardial infarction (MI) increased. The ratio of death due to MI in diabetics to against non-diabetics was 2.6 in Group A, 2.1 in Group B and 4.0 in Group C. CI was found to be twice as common in diabetics in each chronological group, but no remarkable numerical increase was observed in non-diabetics. In diabetics, however, a significant increase in CI was noted in Group B and C in contrast to Group A $(p<0.05)$. There were no remarkable changes in cerebral hemorrhage in either group, while MI showed a significant increase both in diabetics and in nondiabetics, especially in Group $\mathrm{C}(p<0.01)$ as shown in Fig. 1. From anatomical findings, both incidence of CI and MI have previously increased. 
TABLE 2. Causes of death, diabetics Chronological trend-

\begin{tabular}{lrrrrrrr}
\hline & $1953 \sim 62$ & \multicolumn{3}{c}{$1963 \sim 72$} & \multicolumn{2}{c}{$1973 \sim 80$} \\
\cline { 2 - 8 } & Cases & $\%$ & Cases & $\%$ & Cases & $\%$ \\
\hline Diabetic Ketoacidosis & 2 & $(7.4)$ & 0 & $(0.0)$ & 0 & $(0.0)$ \\
Hypoglycemia & 0 & $(0.0)$ & 0 & $(0.0)$ & 1 & $(0.7)$ \\
Macroangiopathy & 7 & $(25.9)$ & 10 & $(23.3)$ & 53 & $(37.9)$ \\
Cerebral infarction & 3 & $(11.1)$ & 5 & $(11.6)$ & 14 & $(10.0)$ \\
Cerebral hemorrhage & 1 & $(3.7)$ & 0 & $(0.0)$ & 10 & $(7.1)$ \\
Myocardial infarction & 2 & $(7.4)$ & 4 & $(9.3)$ & 26 & $(18.6)$ \\
Others (aneurysm etc.) & 1 & $(3.7)$ & 1 & $(2.5)$ & 3 & $(2.1)$ \\
Diabetic nephropathy & 3 & $(11.1)$ & 8 & $(18.6)$ & 23 & $(16.4)$ \\
Malignancy & 3 & $(11.1)$ & 13 & $(30.2)$ & 43 & $(30.7)$ \\
Infections & 12 & $(44.4)$ & 6 & $(14.0)$ & 9 & $(6.4)$ \\
Others & 0 & $(0.0)$ & 6 & $(14.0)$ & 11 & $(7.9)$ \\
\multicolumn{1}{c}{ Total } & 27 & & 43 & & 140 & \\
\hline
\end{tabular}

In order to assess the risk factors for CI and MI, clinical data from prior to the death of the subjects were studied in 65 CI cases and 81 MI cases, and compared to 50 reference cases without CI or MI (Table 4). As shown, the death rate of subjects in the 40-50 group was remarkably lower in the MI and CI groups than in the reference group $(p<0.05)$, but the death rate of subjects in the $70+$ group with MI and CI was significantly higher $(p<0.01)$. There was no differences between the sexes. There are very few subjects with a diabetes duration of

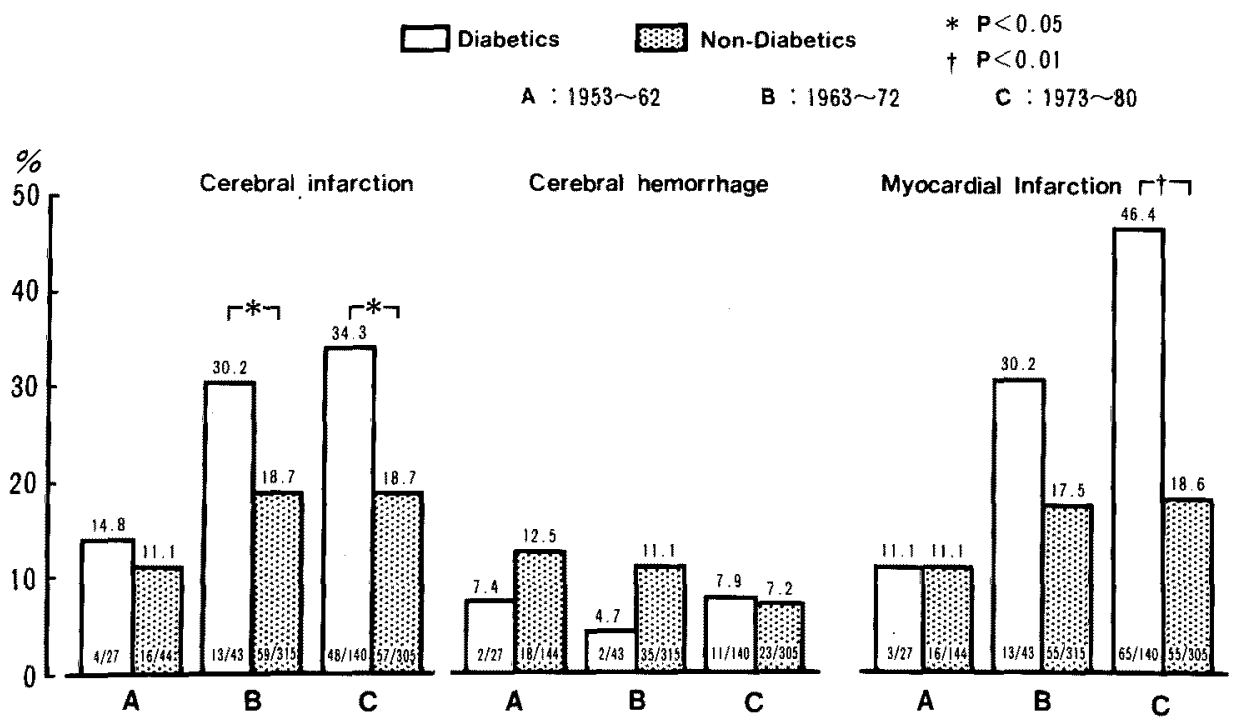

Fig. 1. Morbidity ratio of macroangiopathy in diabetics and non-diabetics, chronological trend. 
TABLE 3. Causes of death, - Non diabetic reference group (743 cases) -

\begin{tabular}{lrrrrrrr}
\hline & \multicolumn{2}{c}{$1953 \sim 62$} & \multicolumn{2}{c}{$1963 \sim 72$} & \multicolumn{2}{c}{$1973 \sim 80$} \\
\cline { 2 - 7 } & \multicolumn{2}{c}{ Case } & $\%$ & Cașes & $\%$ & Cases & $\%$ \\
\hline Vascular diseases & 35 & $(24.3)$ & 71 & $(22.5)$ & 57 & $(18.7)$ \\
Cerebral infarction & 9 & $(6.3)$ & 17 & $(5.4)$ & 18 & $(5.9)$ \\
Cerebral hemorrhage & 16 & $(11.1)$ & 30 & $(9.5)$ & 18 & $(5.9)$ \\
Myocardial infarction & 14 & $(2.8)$ & 14 & $(4.4)$ & 4 & $(4.6)$ \\
Infections & 43 & $(29.9)$ & 48 & $(15.2)$ & 29 & $(9.5)$ \\
Malignancy & 18 & $(12.5)$ & 111 & $(35.2)$ & 144 & $(47.2)$ \\
Others & 29 & $(20.1)$ & 83 & $(26.3)$ & 75 & $(24.6)$ \\
\multicolumn{1}{c}{ Total } & 125 & & 313 & & 305 & \\
\hline
\end{tabular}

TABLE 4. Diabetic picture before death

\begin{tabular}{|c|c|c|c|c|}
\hline \multicolumn{2}{|c|}{ Autopsy findings } & $\begin{array}{c}\text { Non-infactions } \\
n=50(\%)\end{array}$ & $\begin{array}{c}\text { Cerebral } \\
\text { infarction } \\
n=65(\%)\end{array}$ & $\begin{array}{l}\text { Myocardial } \\
\text { infarction } \\
\mathrm{n}=81(\%)\end{array}$ \\
\hline \multirow{4}{*}{ Age at death } & $\sim 49$ yо & 24.0 & $6.2 \dagger$ & $4.9 \dagger$ \\
\hline & $50 \sim 59$ & 32.0 & $15.4^{*}$ & $13.6^{*}$ \\
\hline & $60 \sim 69$ & 24.0 & 29.2 & 33.3 \\
\hline & $70 \sim$ & 20.0 & $49.2 \dagger$ & $48.1 \dagger$ \\
\hline \multicolumn{2}{|l|}{ Sex Male } & 76.0 & 69.2 & 71.6 \\
\hline \multirow{3}{*}{ Duration } & $-5 \mathrm{yrs}$ & 50.0 & $25.4 \dagger$ & $19.2 \dagger$ \\
\hline & $5-10$ & 26.0 & 15.9 & 23.1 \\
\hline & $10 \sim$ & 24.0 & $58.7 \dagger$ & $57.7 \dagger$ \\
\hline \multicolumn{2}{|c|}{ Obese index $>+10 \%$} & 20.5 & 22.6 & 25.5 \\
\hline \multirow{4}{*}{ FBS } & $-100 \mathrm{mg} / 100 \mathrm{ml}$ & 16.3 & 20.0 & 22.4 \\
\hline & -130 & 14.0 & 13.3 & 17.1 \\
\hline & $\sim 150$ & 25.6 & 21.7 & 21.1 \\
\hline & $151 \sim$ & 48.8 & 45.0 & 39.5 \\
\hline \multicolumn{2}{|c|}{ Cholesterol > $230 \mathrm{mg} / 100 \mathrm{ml}$} & 27.3 & $50.9^{*}$ & $53.8 \dagger$ \\
\hline \multicolumn{2}{|c|}{$\mathrm{BP}(160 / 95$ or more $)$} & 15.6 & $45.8 \dagger$ & $33.3^{*}$ \\
\hline \multicolumn{2}{|l|}{${ }^{*} p<0.05$} & & & \\
\hline
\end{tabular}

less than 5 years in the MI and CI groups, whereas a duration of over 10 years was significantly more frequent than in the reference group $(p<0.01)$. Prevalence of obesity and glycemic control were equal in each group. Hypercholesterolemia was more prevalent in the CI group $(p<0.05)$ and especially prevalent in the MI group $(p<0.01)$. Hypertension was also more prevalent in the MI group 


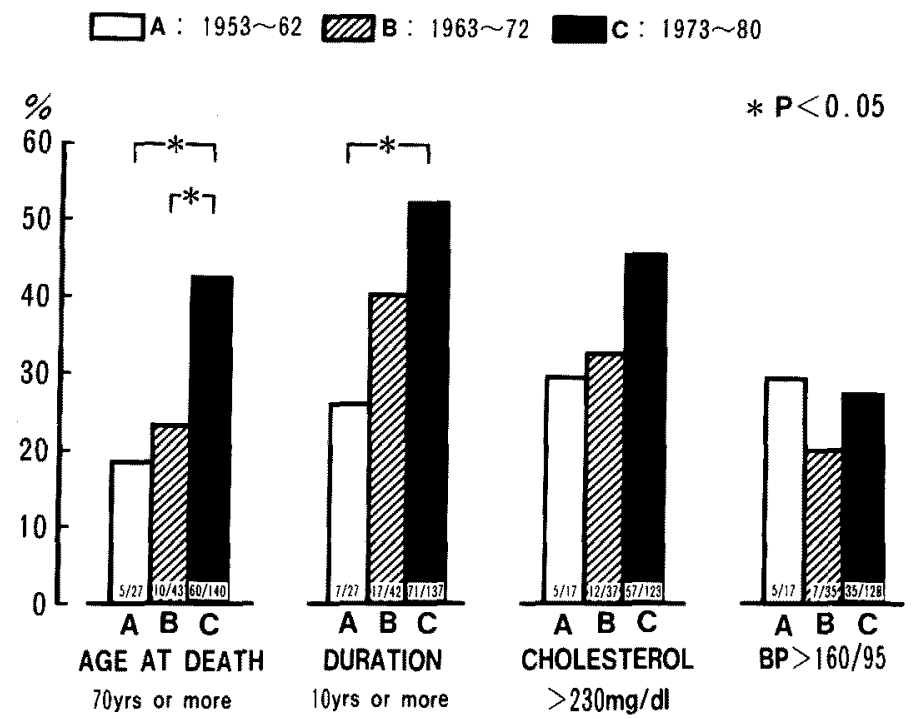

Fig. 2. Diabetic picture before death, chronological trend.

$(p<0.05)$ and in the CI group $(p<0.01)$.

Therefore, the percentage of deaths over the age of 70 , duration of diabetes over 10 years, hypercholesterolemia and hypertension were chronologically compared in diabetic subjects in Groups A, B and C (Fig. 2). In Group C, death over the age of 70 was significantly more common than in the other groups $(p<0.05)$, and duration of over 10 years was also more common than in Group A $(p<0.05)$. But the prevalence of hypercholesterolemia and hypertension did not differ between each group.

Among diabetics who died before the age of 70 , there was a remarkably high percentage of more than 10 year duration in Group $\mathrm{C}$.

\section{Discussion}

The cause of death in Japanese diabetics varied according to the age of death. The statistical report based on anatomical findings concerning the causes of death in diabetics studied between 1958 and 1965, (Goto et al. 1974) stated that the primary cause of death was vascular disease, followed by infectious disease and malignancy. Among vascular diseases, the sequence was as follows: diabetic nephropathy, cerebral vascular disease and coronary heart disease. According to a nationwide survey conducted between 1971 and 1980 (Kosaka and Sakamoto 1981), the most common cause of death in diabetics was vascular disease followed by malignancy, infection and so on. This survey also reported the previous increase in malignancy and vascular diseases.

For vascular disease, our anatomical analysis was again similar to that of Goto. A remarkable decrease in the rate of death from infection and ketoacidosis is thought to be attributable to the recent advances in therapeutic techniques, 
including advances in antibiotics.

In our study, the rate of death due to coronary heart disease appears to have become increasingly significant in diabetics in comparison to cerebrovascular disease. This is possibly the reason why our hospital is located in the southern part of metropolitan Tokyo, and may be a product of the westernization of Tokyoites.

According to statistical reports of Japanese Hawaiian diabetics, the lower incidence of coronary heart disease was not due to racial but environmental factors, especially diet (Kawate et al. 1974, 1976) (Kagan et al. 1972).

It is speculated that more prevalent lesions based on macroangiopathy in Japanese diabetics can be attributed to the numerical increase in elderly diabetics of longer duration. Though hypercholesterolemia was related to MI and CI though not to the recent increase in MI, glycemic control assessed by fasting blood sugar was found to have no direct relation to MI and CI.

Our results are thought to express an increasing trend in complications of diabetes in an increasingly elderly population. This tendency may continue into the future, so measures to solve this problem most be established.

\section{Conclusions}

1) In previous years, the increase in morbidity of coronary heart disease in the diabetic population has resulted in an increased rate of mortality due to MI.

2) During the study period, the ratio of diabetics with CI has risen. However, the number of diabetics whose primary cause of death was CI remained unchanged.

3) Increased morbidity of CI and MI is attributed to the increase in the number of elderly diabetics over the age of 70 with a duration of more than 10 years.

\section{References}

1) Goto, Y., Sato, S. \& Masuda, M. (1974) Causes of death in diabetic autopsy cases. Tohoku J. exp. Med., 112, 339-353.

2) Kagan, A., Harris, B., Winkelstein, W., Johnson, K., Kato, H., Syme, S., Rhoods, G., Gay, M., Nichaman, M., Hamilton, H. \& Tillotson, T. (1972) Epidemiologic studies of coronary heart disease and stroke in Japanese men living in Japan, Hawaii and California. ABCC TR, pp. 16-72.

3) Kawate, R., Miyanishi, M. \& Nishimoto, Y. (1974) Diabetes mellitus in Hawaii. Diabetes J., 2, 112-120.

4) Kawate, R., Miyanishi, M. \& Nishimoto, Y. (1976) Diabetes mellitus in Asia. Int. Congr. Ser., No. 390, Excerpta Medica, Amsterdam, pp. 82-90.

5) Kosaka, K. \& Sakamoto, S. (1981) Diabetics and macroangiopathy. J. Jap. Diab. Soc., 24, 1146-1147. 